\title{
Sexy regulation of SNARE-mediated membrane fusion by local lipid metabolism
}

\author{
Elena Fdez and Sabine Hilfiker* \\ Institute of Parasitology and Biomedicine "López-Neyra," Consejo Superior de Investigaciones Científicas, Granada, Spain \\ *Correspondence: sabine.hilfiker@ipb.csic.es
}

\section{A commentary on}

Sphingosine facilitates SNARE complex assembly and activates synaptic vesicle exocytosis

by Darios, F., Wasser, C., Shakirzyanova, A., Giniatullin, A., Goodman, K., Munoz-Bravo, J. L., Raingo, J., Jorgacevski, J., Kreft, M., Zorec, R., Rosa, J. M., Gandia, L., Gutiérrez, L. M., Binz, T., Giniatullin, R., Kavalali, E. T., and Davletov, B. (2009) Neuron 62, 683-694.

Intracellular vesicle transport processes depend on the ability of membranes to fuse with each other. Such membrane fusion is governed by the soluble $\mathrm{N}$-ethylmaleimide-sensitive factor attachment protein receptor (SNARE) proteins. Neuronal exocytosis requires three SNARE proteins, synaptobrevin-2 (VAMP2) on the synaptic vesicle, and syntaxin-1 and SNAP-25 on the plasma membrane, which assemble into a highly stable ternary complex essential for membrane fusion. Earlier studies have suggested that VAMP2 in synaptic vesicles does not readily interact with syntaxin-1 and SNAP-25 (Hu et al., 2002; Kweon et al., 2003). Such lack of reactivity was attributed to the membrane-proximal part of VAMP2 interacting with the lipid membrane, with positively charged residues interacting with negatively charged phospholipid headgroups, and two tryptophan residues buried in the hydrophobic part of the bilayer (Kweon et al., 2003).

A recent study by Darios et al. (2009) suggests that sphingosine may stimulate the reluctant VAMP2 to engage in fusogenic SNARE complexes. Sphingosine was found to increase SNARE complex formation in vitro, and display a positive effect on neurosecretion in a variety of cellular assays. The sphingosine-mediated enhancement likely involves an increase in the readilyreleasable vesicle pool, implying regulation of a rate-limiting event of neurotransmitter release. Intriguingly, amongst a panel of lipids analysed, the stimulatory effect was specific for sphingosine, indicating that this lipid plays a direct regulatory role in membrane fusion.

This work raises several important questions about the potentiation of SNAREmediated neurosecretion by sphingosine. The first is related to the actual basis upon which the work is built, namely that VAMP2 is not fully available for SNARE complex formation. Indeed, other groups have reported that VAMP2 in proteoliposomes or purified synaptic vesicles readily engages in fusion-competent SNARE complexes, with the rate-limiting factor being the availability of the SNARE acceptor sites on the plasma membrane (Siddiqui et al., 2007; Holt et al., 2008). The reasons for the observed differences in the availability of VAMP2 remain unclear, but possible explanations include experimental differences in the phospholipid composition, proteinlipid ratio and reconstitution procedures of liposomes, as well as differences in vesicle and protein purification protocols. Importantly, whilst vesicular VAMP2 is not available for SNARE complex formation with wildtype recombinant syntaxin-1 and SNAP-25, a certain degree of complex formation was found when using recombinant SNAP-25 devoid of cysteines (Darios et al., 2009), a version of SNAP-25 used in studies demonstrating free availability of VAMP2 (Siddiqui et al., 2007). Thus, the distinct types of SNAP-25 employed may account for at least part of the observed discrepancies. In either case, it will be interesting to see whether sphingosine has positive modulatory effects upon the kinetics of SNARE complex formation even under conditions in which VAMP2 seems to be freely available.

The second question relates to VAMP2 being a specific target for sphingosine. The best evidence for this comes from observations that sphingosine no longer potentiates secretion in VAMP2-null mutant neurons (Darios et al., 2009). However, it remains formally possible that the chronic absence of VAMP2 is associated with changes in upstream events leading to SNARE complex formation upon which sphingosine normally acts. Alternative approaches, such as assessing the effects of sphingosine in neurons upon transient knockdown of VAMP2 by RNAi approaches, or in synapses expressing VAMP2 mutants (Maximov et al., 2009) may help to further evaluate specificity of sphingosine action with respect to VAMP2. In either case, it seems likely that exogenous sphingosine will induce additional changes in membrane fluidity and curvature which may impact upon vesicle fusion in a VAMP2independent manner.

Assuming that the availability of VAMP2 for SNARE complex formation is limited, the third question arising from the work by Darios et al. (2009) is related to exactly how sphingosine may regulate the availability of VAMP2. The membrane-proximal region of VAMP2 contains a cluster of basic residues thought to interact with the negatively charged phospholipid headgroups, and an amphipathic weak base such as sphingosine may interfere with this interaction. If this were the case, decreasing the net positive charge of the membrane-proximal region of VAMP2 would be expected to enhance SNARE complex formation. However, mutants which progressively decrease the overall net positive charge of this region of VAMP2 seem to decrease, rather than increase, vesicular secretion (Williams et al., 2009). These data have to be interpreted with care, as a reduction in evoked release may be the result of increased spontaneous vesicle fusion (i.e. due to a run-down of the readily-releasable pool). Alternatively, extensive mutations may alter the secondary structure of VAMP2 in this region. Indeed, recent structural data on membrane-bound VAMP2 indicate the existence of substantial secondary 
structure, including a novel N-terminal motif helix (Ellena et al., 2009). Whilst this motif has been proposed to speed up subsequent SNARE complex formation (Ellena et al., 2009), the availability of such structured lipid-bound VAMP2 for SNARE complex formation remains to be determined, and dynamic structural changes induced by the presence of sphingosine remain possible.

Irrespective of target specificity, the study by Darios et al. (2009) indicates that local lipid metabolism can rapidly and profoundly affect neurotransmission. Whilst the stimulatory effect on release seems to mainly involve an increase in the readily-releasable vesicle pool, sphingosine may have multiple and even opposing roles in regulating secretion events, dependent on the pool of vesicles under study. Sphingosine effects on neurosecretion may further vary with exposure time, with a transient increase leading to enhanced transmitter release (Darios et al., 2009) but a more chronic increase leading to decreased release (Camoletto et al., 2009). In addition, sphingosine is likely to have additional effects on vesicle pool turnover. For example, it will be interesting to determine whether sphingosine can modulate SNARE complex disassembly, recently described to be facilitated by the membrane interaction of the NSF co-factor $\alpha$-SNAP (Winter et al., 2009), or whether it plays post-fusion roles in the recycling of VAMP2 upon its dissociation from the
SNARE complex. Additional vesicular fusion events may be affected by sphingosine as well, since closely related VAMP2 isoforms involved in other intracellular membrane trafficking also possess the positively charged juxtamembrane region proposed to be subject to possible regulation by lipid interactions.

Finally, since modulating the levels of sphingosine and its metabolites is considered a valid novel strategy in treating a variety of neurodegenerative diseases such as Alzheimer's, Niemann-Pick disease or multiple sclerosis, the link between sphingosine and regulation of vesicular trafficking events described by Darios et al. (2009) may help in the description of new molecular disease targets and pathways.

\section{REFERENCES}

Camoletto, P. G., Vara, H., Morando, L., Connell, E., Marletto, F. P., Giustetto, M., Sassoe-Pognetto, M. Van Veldhoven, P. P., and Ledesma, M. D. (2009). Synaptic vesicle docking: sphingosine regulates syntaxin1 interaction with Munc18. Plos ONE, 4, e5310. doi:10.1371/journal.pone.0005310.

Darios, F., Wasser, C., Shakirzyanova, A., Giniatullin, A., Goodman, K., Munoz-Bravo, J. L., Raingo, J., Jorgacevski, J., Kreft, M., Zorec, R., Rosa, J. M., Gandia, L., Gutiérrez, L. M., Binz, T., Giniatullin, R., Kavalali, E. T., and Davletov, B. (2009). Sphingosine facilitates SNARE complex assembly and activates synaptic vesicle exocytosis. Neuron 62, 683-694.

Ellena, J. F., Liang, B., Wiktor, M., Stein, A., Cafiso, D. S., Jahn, R., and Tamm, L. K. (2009). Dynamic structure of lipid-bound synaptobrevin suggests a nucleation-propagation mechanism for trans-SNARE complex formation. Proc. Natl. Acad. Sci. USA 106, 20306-20311.
Holt, M., Riedel, D., Stein, A., Schuette, C., and Jahn, R. (2008). Synaptic vesicles are constitutively active fusion machines that function independently of $\mathrm{Ca}^{2+}$. Curr. Biol. 18, 715-722.

Hu, K., Carroll, J., Fedorovich, S., Rickman, C., Sukhodub, A. and Davletov, B. (2002). Vesicular restriction of synaptobrevin suggests a role for calcium in membrane fusion. Nature 415, 646-650.

Kweon,D.H., Kim, C.S., and Shin, Y. K. (2003). Regulation of neuronal SNARE assembly by the membrane. Nat. Struct. Biol. 10, 440-447.

Maximov, A., Tang, J., Yang, X., Pang, Z. P., and Südhof, T. C. (2009). Complexin controls the force transfer from SNARE complexes to membranes in fusion. Science 323, 516-521.

Siddiqui, T. J., Vites, O., Stein, A., Heintzmann, R., Jahn, R., and Fasshauer, D. (2007). Determinants of synaptobrevin regulation in membranes. Mol. Biol. Cell 18, 2037-2046.

Williams, D., Vicogne, J., Zaitseva, I., McLaughlin, S., and Pessin, J.E. (2009). Evidence that electrostatic interactions between vesicle-associated membrane protein 2 and acidic phospholipids may modulate the fusion of transport vesicles with the plasma membrane. $\mathrm{Mol}$. Biol. Cell 20, 4910-4919.

Winter, U., Chen, X., and Fasshauer, D. (2009). A conserved membrane attachment site in $\alpha$-SNAP facilitates N-ethylmaleimide-sensitive factor (NSF)-driven SNARE complex disassembly. J. Biol. Chem. 284, 31817-31826

Received: 24 February 2010; accepted: 29 March 2010; published online: 25 June 2010.

Citation: Fdez E and Hilfiker S (2010) Sexy regulation of SNARE-mediated membrane fusion by local lipid metabolism. Front. Syn. Neurosci. 2:3. doi: 10.3389/fnsyn.2010.00003

Copyright $(2010$ Fdez and Hilfiker. This is an open-access article subject to an exclusive license agreement between the authors and the Frontiers Research Foundation, which permits unrestricted use, distribution, and reproduction in any medium, provided the original authors and source are credited. 